\title{
EXPLICIT DECOMPOSITION OF A RATIONAL PRIME IN A CUBIC FIELD
}

\author{
ŞABAN ALACA, BLAIR K. SPEARMAN, AND KENNETH S. WILLIAMS
}

Received 19 June 2005; Revised 19 February 2006; Accepted 12 March 2006

We give the explicit decomposition of the principal ideal $\langle p\rangle$ ( $p$ prime) in a cubic field.

Copyright (c) 2006 Hindawi Publishing Corporation. All rights reserved.

\section{Introduction}

Let $K$ be an algebraic number field. Let $O_{K}$ denote the ring of integers of $K$. Let $d(K)$ denote the discriminant of $K$. Let $\theta \in O_{K}$ be such that $K=\mathbb{Q}(\theta)$. The minimal polynomial of $\theta$ over $\mathbb{Q}$ is denoted by $\operatorname{irr}_{\mathbb{Q}}(\theta)$. The discriminant $D(\theta)$ and the index $\operatorname{ind}(\theta)$ of $\theta$ are related by the equation

$$
D(\theta)=(\operatorname{ind}(\theta))^{2} d(K)
$$

If $p$ is a prime not dividing ind $(\theta)$, then it is well known that the following theorem of Dedekind gives explicitly the factorization of the principal ideal $\langle p\rangle$ of $O_{K}$ into prime ideals in terms of the irreducible factors of $\operatorname{irr}_{\mathbb{Q}}(\theta)$ modulo $p$; see, for example, [3, Theorem 10.5.1, page 257].

Theorem 1.1. Let $K=\mathbb{Q}(\theta)$ be an algebraic number field with $\theta \in O_{K}$. Let $p$ be a rational prime. Let

$$
f(x)=\operatorname{irr}_{\mathbb{Q}}(\theta) \in \mathbb{Z}[x] .
$$

Let ${ }^{-}$denote the natural map $\mathbb{Z}[x] \rightarrow \mathbb{Z}_{p}[x]$, where $\mathbb{Z}_{p}=\mathbb{Z} / p \mathbb{Z}$. Let

$$
\bar{f}(x)=g_{1}(x)^{e_{1}} \cdots g_{r}(x)^{e_{r}},
$$

where $g_{1}(x), \ldots, g_{r}(x)$ are distinct irreducible polynomials in $\mathbb{Z}_{p}[x]$, and $e_{1}, \ldots, e_{r}$ are positive 
integers. For $i=1,2, \ldots, r$, let $f_{i}(x)$ be any polynomial of $\mathbb{Z}[x]$ such that $\bar{f}_{i}=g_{i}$ and $\operatorname{deg}\left(f_{i}\right)=$ $\operatorname{deg}\left(g_{i}\right)$. Set

$$
P_{i}=\left\langle p, f_{i}(\theta)\right\rangle, \quad i=1,2, \ldots, r
$$

If $\operatorname{ind}(\theta) \not \equiv 0(\bmod p)$, then $P_{1}, \ldots, P_{r}$ are distinct prime ideals of $O_{K}$ with

$$
\begin{gathered}
\langle p\rangle=P_{1}^{e_{1}} \cdots P_{r}^{e_{r}}, \\
N\left(P_{i}\right)=p^{\operatorname{deg} f_{i}}, \quad i=1,2, \ldots, r .
\end{gathered}
$$

On the other hand if $p$ is a prime dividing ind $(\theta)$, no such general theorem is known which gives the prime ideals explicitly, and all that is available in general is the BuchmannLenstra algorithm [4, page 315] for decomposing a prime in a number field. If $p$ is not a common index divisor of $K$, then there exist elements $\phi \in O_{K}$ for which $K=\mathbb{Q}(\phi)$, and $p \nmid \operatorname{ind}(\phi)$, and we can apply Dedekind's theorem to obtain the prime ideal factorization of $\langle p\rangle$ from the minimal polynomial $\operatorname{irr}_{\mathbb{Q}}(\phi)$. However given $\theta$ it is not easy to determine such an element $\phi$ in general. Moreover when $p$ is a common index divisor of $K$, no such element $\phi$ exists and Dedekind's theorem cannot be applied.

In this paper we treat the case when $K$ is a cubic field and $p$ is a prime dividing ind $(\theta)$. When $p$ is a common index divisor of $K$ (the only possibility is $p=2$ ), we quote the results in [2]. When $p$ is not a common index divisor, we construct an element $\phi \in O_{K}$ such that $K=\mathbb{Q}(\phi)$ and $p \nmid \operatorname{ind}(\phi)$ and then apply Dedekind's theorem to obtain the prime ideal factorization of $\langle p\rangle$. Our construction of $\phi$ was guided by the $p$-integral bases of $K$ given by Alaca [1]. We give explicitly the prime ideals in the factorization of $\langle p\rangle$ into prime ideals in $O_{K}$. The form of the prime ideal factorization has been given by Llorente and Nart [6, Theorem 1, page 580] and we make use of their results. A method for factoring all primes in a cubic field is given in [5, pages 119-121]. It is well known that $K$ can be given in the form $K=\mathbb{Q}(\theta)$, where $\theta$ is a root of the irreducible polynomial

$$
f(x)=x^{3}-a x+b, \quad a, b \in \mathbb{Z},
$$

so that $\operatorname{irr}_{\mathbb{Q}}(\theta)=f(x)$. Moreover it is further known that $a$ and $b$ can be chosen so that there are no primes $p$ with $p^{2} \mid a$ and $p^{3} \mid b$. We have

$$
D(\theta)=4 a^{3}-27 b^{2}
$$

Let $v_{p}(k)$ denote the largest nonnegative integer $m$ such that $p^{m}$ divides the nonzero integer $k$. From (1.1) we deduce that

$$
v_{p}(\operatorname{ind}(\theta))=\frac{v_{p}(D(\theta))-v_{p}(d(K))}{2} .
$$

We set

$$
D_{p}(\theta)=\frac{D(\theta)}{p^{\nu_{p}(D(\theta))}} .
$$


Table 1.1. $p=2$.

\begin{tabular}{|c|c|c|c|c|c|c|c|}
\hline Case & Conditions & $v_{2}(d(K))$ & $\nu_{2}(\operatorname{ind}(\theta))$ & $\phi$ & $\begin{array}{l}\text { Factors } \\
\text { of }\langle 2\rangle\end{array}$ & $\begin{array}{l}\text { Prime } \\
\text { ideals }\end{array}$ & Norms \\
\hline Al & $\begin{array}{l}a \equiv 0(4), b \equiv 4(8) \\
v_{2}(D(\theta))=4\end{array}$ & 2 & 1 & $\phi=\frac{\theta^{2}}{2}$ & $P^{3}$ & $P=\langle 2, \phi\rangle$ & $N(P)=2$ \\
\hline A2 & $\begin{array}{l}a \equiv 2(4), b \equiv 0(8) \\
v_{2}(D(\theta))=5\end{array}$ & 3 & 1 & $\phi=1+\theta+\frac{\theta^{2}}{2}$ & $P Q^{2}$ & $\begin{array}{l}P=\langle 2,1+\phi\rangle \\
Q=\langle 2, \phi\rangle\end{array}$ & $\begin{array}{l}N(P)=2 \\
N(Q)=2\end{array}$ \\
\hline A3 & $\begin{array}{l}a \equiv 2(4), b \equiv 4(8) \\
v_{2}(D(\theta))=4\end{array}$ & 2 & 1 & $\phi=\frac{\theta^{2}}{2}$ & $P Q^{2}$ & $\begin{array}{l}P=\langle 2, \phi\rangle \\
Q=\langle 2,1+\phi\rangle\end{array}$ & $\begin{array}{l}N(P)=2 \\
N(Q)=2\end{array}$ \\
\hline A4 & $\begin{array}{l}a \equiv 1(4), b \equiv 0(4) \\
D_{2}(\theta) \equiv 1(8) \\
v_{2}(D(\theta))=2\end{array}$ & 0 & 1 & $\phi=\frac{\theta+\theta^{2}}{2}$ & $P Q R$ & $\begin{array}{l}P=\langle 2, \theta\rangle \\
Q=\langle 2,1+\phi\rangle \\
R=\langle 2,1+\theta+\phi\rangle\end{array}$ & $\begin{array}{l}N(P)=2 \\
N(Q)=2 \\
N(R)=2\end{array}$ \\
\hline A5 & \begin{tabular}{|l}
$a \equiv 1(4), b \equiv 0(4)$ \\
$D_{2}(\theta) \equiv 5(8)$ \\
$v_{2}(D(\theta))=2$
\end{tabular} & 0 & 1 & $\phi=\frac{\theta+\theta^{2}}{2}$ & $P Q$ & $\begin{array}{l}P=\langle 2, \phi\rangle \\
Q=\left\langle 2,1+\phi+\phi^{2}\right\rangle\end{array}$ & $\begin{array}{l}N(P)=2 \\
N(Q)=4\end{array}$ \\
\hline A6 & $\begin{array}{l}a \equiv 3(4), b \equiv 2(4) \\
v_{2}(D(\theta)) \equiv 1(2) \\
v_{2}(D(\theta)) \geq 5\end{array}$ & 3 & $\frac{\nu_{2}(D(\theta))-3}{2}$ & $\begin{array}{l}\phi=1+\lambda+\frac{\lambda^{2}}{2} \\
\lambda=\frac{\alpha}{2^{m+1}} \\
m=\frac{\nu_{2}(D(\theta))-3}{2}\end{array}$ & $P Q^{2}$ & $\begin{array}{l}P=\langle 2,1+\phi\rangle \\
Q=\langle 2, \phi\rangle\end{array}$ & $\begin{array}{l}N(P)=2 \\
N(Q)=2\end{array}$ \\
\hline A7 & $\begin{array}{l}a \equiv 3(4), b \equiv 2(4) \\
v_{2}(D(\theta)) \equiv 0(2) \\
v_{2}(D(\theta)) \geq 4 \\
D_{2}(\theta) \equiv 3(4)\end{array}$ & 2 & $\frac{\nu_{2}(D(\theta))-2}{2}$ & $\begin{array}{l}\phi=\frac{\alpha}{2^{m+1}} \\
m=\frac{\nu_{2}(D(\theta))-2}{2}\end{array}$ & $P Q^{2}$ & $\begin{array}{l}P=\langle 2, \phi\rangle \\
Q=\langle 2,1+\phi\rangle\end{array}$ & $\begin{array}{l}N(P)=2 \\
N(Q)=2\end{array}$ \\
\hline A8 & \begin{tabular}{|l}
$a \equiv 3(4), b \equiv 2(4)$ \\
$v_{2}(D(\theta)) \equiv 0(2)$ \\
$v_{2}(D(\theta)) \geq 4$ \\
$D_{2}(\theta) \equiv 1(8)$
\end{tabular} & 0 & $\frac{v_{2}(D(\theta))}{2}$ & $\begin{array}{l}\phi=\frac{\alpha}{2^{m}} \\
m=\frac{\nu_{2}(D(\theta))}{2}\end{array}$ & $P Q R$ & $\begin{array}{l}P=\langle 2, \phi\rangle \\
Q=\left\langle 2, \frac{2+\phi+\phi^{2}}{2}\right\rangle \\
R=\left\langle 2, \frac{2+3 \phi+\phi^{2}}{2}\right\rangle\end{array}$ & $\begin{array}{l}N(P)=2 \\
N(Q)=2 \\
N(R)=2\end{array}$ \\
\hline A9 & $\begin{array}{l}a \equiv 3(4), b \equiv 2(4) \\
v_{2}(D(\theta)) \equiv 0(2) \\
v_{2}(D(\theta)) \geq 4 \\
D_{2}(\theta) \equiv 5(8)\end{array}$ & 0 & $\frac{\nu_{2}(D(\theta))}{2}$ & $\begin{array}{l}\phi=\frac{\lambda+\lambda^{2}}{2} \\
\lambda=\frac{\alpha}{2^{m+1}} \\
m=\frac{\nu_{2}(D(\theta))-2}{2}\end{array}$ & $P Q$ & $\begin{array}{l}P=\langle 2, \phi\rangle \\
Q=\left\langle 2,1+\phi+\phi^{2}\right\rangle\end{array}$ & $\begin{array}{l}N(P)=2 \\
N(Q)=4\end{array}$ \\
\hline
\end{tabular}

The determination of $\nu_{p}(d(K))$ was carried out by Llorente and Nart [6, Theorem 2, page 583 ] in 1983; see also Alaca [1]. The values of $\nu_{p}(D(\theta))$ and $\nu_{p}(d(K))$ are listed in tabular form in Alaca [1] depending on congruence conditions on $a$ and $b$. From [1] we deduce that $p \mid \operatorname{ind}(\theta)$ in precisely those cases listed in Tables 1.1, 1.2, 1.3, and no others. We abbreviate $r \equiv s(\bmod m)$ by $r \equiv s(m)$. In the sixth column of each table we give the form of the prime ideal factorization from the work of Llorente and Nart [6, Theorem 1, page 580]. However, Llorente and Nart did not give the prime ideals explicitly. We give explicit formulae for these prime ideals in the seventh column of each of Tables 1.1, 1.2, and 1.3. It is convenient to set

$$
\alpha=-4 a^{2}+9 b \theta+6 a \theta^{2} \in O_{K} .
$$




\section{Decomposition of primes in a cubic field}

Table 1.2. $p=3$.

\begin{tabular}{|c|c|c|c|c|c|c|c|}
\hline Case & Conditions & $\nu_{3}(d(K))$ & $\nu_{3}(\operatorname{ind}(\theta))$ & $\phi$ & $\begin{array}{l}\text { Factors } \\
\text { of }\langle 3\rangle\end{array}$ & $\begin{array}{l}\text { Prime } \\
\text { ideals }\end{array}$ & Norms \\
\hline B1 & $\begin{array}{c}2=v_{3}(b) \\
=v_{3}(a) \\
v_{3}(D(\theta))=6\end{array}$ & 4 & 1 & $\phi=\frac{\theta^{2}}{3}$ & $P^{3}$ & $P=\langle 3, \phi\rangle$ & $N(P)=3$ \\
\hline B2 & $\begin{array}{c}2=v_{3}(b) \\
<v_{3}(a) \\
v_{3}(D(\theta))=7\end{array}$ & 5 & 1 & $\phi=\frac{\theta^{2}}{3}$ & $P^{3}$ & $P=\langle 3, \phi\rangle$ & $N(P)=3$ \\
\hline B3 & $\begin{array}{c}1=\nu_{3}(a) \\
<\nu_{3}(b) \\
\nu_{3}(D(\theta))=3\end{array}$ & 1 & 1 & 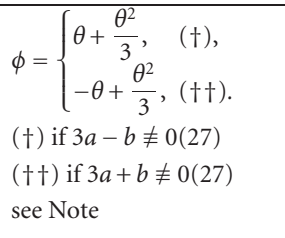 & $P Q^{2}$ & $\begin{array}{l}P=\langle 3, \phi\rangle \\
Q=\left\langle 3, \phi-\frac{a}{3}\right\rangle\end{array}$ & $\begin{array}{l}N(P)=3 \\
N(Q)=3\end{array}$ \\
\hline B4 & $\begin{array}{l}\nu_{3}(a) \geq 1 \\
\nu_{3}(b)=0 \\
a \neq 3(9) \\
b^{2} \equiv a+1(9) \\
\nu_{3}(D(\theta))=3\end{array}$ & 1 & 1 & 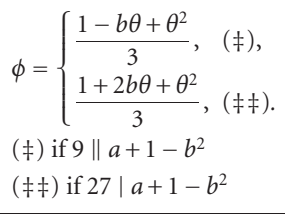 & $P Q^{2}$ & $\begin{array}{l}P=\left\langle 3, \frac{-(2 a+3)}{3}+\phi\right\rangle \\
Q=\langle 3, \phi\rangle,(\ddagger) \\
P=\left\langle 3, \frac{a}{3}+\phi\right\rangle \\
Q=\langle 3,1+\phi\rangle,(\neq \neq)\end{array}$ & $\begin{array}{l}N(P)=3 \\
N(Q)=3\end{array}$ \\
\hline B5 & $\begin{array}{l}a \equiv 3(9) \\
v_{3}(b)=0 \\
b^{2} \equiv 4(9) \\
b^{2} \equiv a+1(27) \\
v_{3}(D(\theta))=5\end{array}$ & 3 & 1 & $\phi=\frac{1-b \theta+\theta^{2}}{3}$ & $P^{3}$ & $P=\langle 3, \phi\rangle$ & $N(P)=3$ \\
\hline B6 & $\begin{array}{l}a \equiv 3(9) \\
v_{3}(b)=0 \\
b^{2} \equiv a+1(27) \\
v_{3}(D(\theta)) \equiv 1(2) \\
v_{3}(D(\theta)) \geq 7\end{array}$ & ) & $\frac{v_{3}(D(\theta))-1}{2}$ & $\begin{array}{l}\phi=\left\{\begin{array}{l}-\lambda+\frac{\lambda^{2}}{3}, \\
\lambda+\frac{\lambda^{2}}{3},\end{array},(* *)\right. \\
\lambda=\frac{\alpha}{3^{m+2}} \\
m=\frac{\nu_{3}(D(\theta))-3}{2} \\
(*) \text { if } a \neq-3^{m-1} D_{3}(\theta)(9) \\
(* *) \text { if } a \neq 3^{m-1} D_{3}(\theta)(9) \\
\text { see Note }\end{array}$ & $P Q^{2}$ & $\begin{array}{l}P=\langle 3, \phi\rangle \\
Q=\left\langle 3, \phi-\frac{a D_{3}(\theta)}{3}\right\rangle\end{array}$ & $\begin{array}{l}N(P)=3 \\
N(Q)=3\end{array}$ \\
\hline B7 & $\begin{array}{l}a \equiv 3(9) \\
v_{3}(b)=0 \\
b^{2} \equiv a+1(27) \\
v_{3}(D(\theta)) \equiv 0(2) \\
v_{3}(D(\theta)) \geq 6 \\
D_{3}(\theta) \equiv 2(3)\end{array}$ & ) & $\frac{\nu_{3}(D(\theta))}{2}$ & $\begin{aligned} \phi & =\frac{\alpha}{3^{m+2}} \\
m & =\frac{\nu_{3}(D(\theta))-2}{2}\end{aligned}$ & $P Q$ & $\begin{aligned} P= & \langle 3,2+\phi\rangle \\
Q= & \left\langle 3,2+\phi+\phi^{2}\right\rangle \\
& \text { if } m=2, \\
P= & \langle 3, \phi\rangle \\
Q= & \left\langle 3,1+\phi^{2}\right\rangle \\
& \text { if } m \geq 3\end{aligned}$ & $\begin{array}{l}N(P)=3 \\
N(Q)=9\end{array}$ \\
\hline B8 & $\begin{array}{l}a \equiv 3(9) \\
v_{3}(b)=0 \\
b^{2} \equiv a+1(27) \\
v_{3}(D(\theta)) \equiv 0(2) \\
v_{3}(D(\theta))=6 \\
D_{3}(\theta) \equiv 1(3)\end{array}$ & 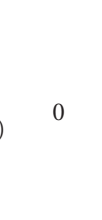 & 3 & & $P$ & $P=\langle 3\rangle$ & $N(P)=27$ \\
\hline
\end{tabular}


Şaban Alaca et al. 5

Table 1.2. Continued.

\begin{tabular}{|c|c|c|c|c|c|c|c|}
\hline Case & Conditions & $v_{3}(d(K))$ & $\nu_{3}(\operatorname{ind}(\theta))$ & $\phi$ & $\begin{array}{l}\text { Factors } \\
\text { of }\langle 3\rangle\end{array}$ & $\begin{array}{l}\text { Prime } \\
\text { ideals }\end{array}$ & Norms \\
\hline B9 & $\begin{array}{l}a \equiv 3(9) \\
v_{3}(b)=0 \\
b^{2} \equiv a+1(27) \\
v_{3}(D(\theta)) \equiv 0(2) \\
v_{3}(D(\theta)) \geq 8 \\
D_{3}(\theta) \equiv 1(3)\end{array}$ & 0 & $\frac{\nu_{3}(D(\theta))}{2}$ & $\begin{aligned} \phi & =\frac{\alpha}{3^{m+2}} \\
m & =\frac{\nu_{3}(D(\theta))-2}{2}\end{aligned}$ & $P Q R$ & $\begin{array}{l}P=\langle 3, \phi\rangle \\
Q=\langle 3,-1+\phi\rangle \\
R=\langle 3,1+\phi\rangle\end{array}$ & $\begin{array}{l}N(P)=3 \\
N(Q)=3 \\
N(R)=3\end{array}$ \\
\hline
\end{tabular}

Note: In case B3 (resp., B6) if $b \equiv 0$ (27) (resp., $m \geq 3$ ), both choices for $\phi$ are valid.

Table 1.3. $p>3$.

\begin{tabular}{|c|c|c|c|c|c|c|c|}
\hline Case & Conditions & $v_{p}(d(K))$ & $v_{p}(\operatorname{ind}(\theta))$ & $\phi$ & $\begin{array}{l}\text { Factors } \\
\text { of }\langle p\rangle\end{array}$ & $\begin{array}{l}\text { Prime } \\
\text { ideals }\end{array}$ & Norms \\
\hline $\mathrm{C} 1$ & $\begin{array}{l}2=v_{p}(b) \leq v_{p}(a) \\
v_{p}(D(\theta))=4\end{array}$ & 2 & 1 & $\phi=\frac{\theta^{2}}{p}$ & $P^{3}$ & $P=\langle p, \phi\rangle$ & $N(P)=p$ \\
\hline C2 & $\begin{array}{l}1=v_{p}(a)<v_{p}(b) \\
v_{p}(D(\theta))=3\end{array}$ & 1 & 1 & $\phi= \begin{cases}\frac{\theta^{2}}{p}, & \text { if } p^{2} \| b \\
\theta+\frac{\theta^{2}}{p}, & \text { if } p^{3} \mid b\end{cases}$ & $P Q^{2}$ & $\begin{array}{l}P=\langle p, \phi\rangle \\
Q=\left\langle p,-\frac{a}{p}+\phi\right\rangle\end{array}$ & $\begin{array}{l}N(P)=p \\
N(Q)=p\end{array}$ \\
\hline C3 & $\begin{array}{l}v_{p}(a)=v_{p}(b)=0 \\
v_{p}(D(\theta)) \equiv 1(2) \\
v_{p}(D(\theta)) \geq 3\end{array}$ & 1 & $\frac{v_{p}(D(\theta))-1}{2}$ & $\begin{array}{l}\phi=\lambda+\frac{\lambda^{2}}{p} \\
\lambda=\frac{\alpha}{p^{m}} \\
m=\frac{v_{p}(D(\theta))-1}{2}\end{array}$ & $P Q^{2}$ & $\begin{array}{l}P=\langle p, \phi\rangle \\
Q=\left\langle p,-3 a D_{p}(\theta)+\phi\right\rangle\end{array}$ & $\begin{array}{l}N(P)=p \\
N(Q)=p\end{array}$ \\
\hline $\mathrm{C} 4$ & $\begin{array}{l}v_{p}(a)=v_{p}(b)=0 \\
v_{p}(D(\theta)) \equiv 0(2) \\
v_{p}(D(\theta)) \geq 2 \\
\left(\frac{D_{p}(\theta)}{p}\right)=1\end{array}$ & 0 & $\frac{v_{p}(D(\theta))}{2}$ & $\begin{aligned} \phi & =\frac{\alpha}{p^{m}} \\
m & =\frac{v_{p}(D(\theta))}{2} \\
t^{2} & \equiv 3 a D_{p}(\theta)(p)\end{aligned}$ & $P Q R$ & $\begin{array}{l}P=\langle p, \phi\rangle \\
Q=\langle p,-t+\phi\rangle \\
R=\langle p, t+\phi\rangle\end{array}$ & $\begin{array}{l}N(P)=p \\
N(Q)=p \\
N(R)=p\end{array}$ \\
\hline C5 & $\begin{array}{l}v_{p}(a)=v_{p}(b)=0 \\
v_{p}(D(\theta)) \equiv 0(2) \\
v_{p}(D(\theta)) \geq 2 \\
\left(\frac{D_{p}(\theta)}{p}\right)=-1\end{array}$ & 0 & $\frac{v_{p}(D(\theta))}{2}$ & $\begin{array}{l}\phi=\frac{\alpha}{p^{m}} \\
m=\frac{v_{p}(D(\theta))}{2}\end{array}$ & $P Q$ & $\begin{array}{l}P=\langle p, \phi\rangle \\
Q=\left\langle p,-3 a D_{p}(\theta)+\phi^{2}\right\rangle\end{array}$ & $\begin{array}{l}N(P)=p \\
N(Q)=p^{2}\end{array}$ \\
\hline
\end{tabular}

It is easy to show that the minimal polynomial of $\alpha$ over $\mathbb{Q}$ is

$$
q(x)=x^{3}-3 a D(\theta) x+D(\theta)^{2}
$$

and that

$$
\operatorname{disc}(q(x))=3^{6} b^{2} D(\theta)^{3} .
$$




\section{Case A1}

In this case we can define integers $A$ and $B$ by $a=4 A$ and $b=8 B+4$. Set $\phi=\theta^{2} / 2$. The minimal polynomial of $\phi$ over $\mathbb{Q}$ is

$$
p(x)=x^{3}-4 A x^{2}+4 A^{2} x-\left(8 B^{2}+8 B+2\right)
$$

so that $\phi \in O_{K}$. Further

$$
\operatorname{disc}(p(x))=-4(2 B+1)^{2}\left(108 B^{2}+108 B-16 A^{3}+27\right) .
$$

We have $p(x) \equiv x^{3}(\bmod 2)$. As $2^{2}\left\|\operatorname{disc}(p(x)), 2^{2}\right\| d(K)$, we have $2 \nmid \operatorname{ind}(\phi)$, so that by Theorem 1.1,

$$
\langle 2\rangle=\langle 2, \phi\rangle^{3} \text {. }
$$

\section{Cases A2, A3, A5, A7, B1, B2, B5, B7, B9, C1, C2}

These cases can be treated similarly to case A1.

\section{Cases A4, A8}

In these cases 2 is a common index divisor and we can appeal to [6, Theorem 4, page 585] for the results.

\section{Case A6}

We let $\lambda=\alpha / 2^{m+1}$, where $\nu_{2}(D(\theta))=2 m+3 \geq 5$, and $\phi=1+\lambda+\lambda^{2} / 2$. By (1.11), the minimal polynomial of $\alpha$ over $\mathbb{Q}$ is $x^{3}-3 a D(\theta) x+D(\theta)^{2}$ so that the minimal polynomial of $\lambda$ over $\mathbb{Q}$ is

$$
x^{3}-\frac{3 a D(\theta)}{2^{2 m+2}} x+\frac{D(\theta)^{2}}{2^{3 m+3}}=x^{3}-6 a D_{2}(\theta) x+2^{m+3} D_{2}(\theta)^{2} .
$$

Hence $\lambda \in O_{K}$. We are now in case A2 with

$$
\begin{gathered}
a^{\prime}=6 a D_{2}(\theta) \equiv 2(\bmod 4), \\
b^{\prime}=2^{m+3} D_{2}(\theta)^{2} \equiv 0(\bmod 8), \\
D(\theta)^{\prime}=\frac{3^{6} b^{2} D(\theta)^{3}}{2^{6 m+6}}, \\
v_{2}(D(\theta))^{\prime}=2+3(2 m+3)-(6 m+6)=5 .
\end{gathered}
$$

Thus by case A2 we obtain

$$
\langle 2\rangle=\langle 2, \phi+1\rangle\langle 2, \phi\rangle^{2}
$$




\section{Case A9}

In this case we set $\nu_{2}(D(\theta))=2 m+2$ (so that $\left.m \geq 1\right), \lambda=\alpha / 2^{m+1}$, and $\phi=\left(\lambda+\lambda^{2}\right) / 2$. Then proceeding as in case A6 we can reduce this case to case A5.

\section{Case B3}

In this case we have

$$
1=\nu_{3}(a)<\nu_{3}(b), \quad \nu_{3}(D(\theta))=3 .
$$

Clearly $9 \mid 3 a-b$ and $9 \mid 3 a+b$. However 27 cannot divide both of $3 a-b$ and $3 a+b$ as their sum $6 a$ is not divisible by 27 . Hence we can define

$$
\phi= \begin{cases}\frac{\theta^{2}}{3}+\theta & \text { if } 3 a-b \not \equiv 0(\bmod 27), \\ \frac{\theta^{2}}{3}-\theta & \text { if } 3 a+b \neq \equiv(\bmod 27) .\end{cases}
$$

We note that if $27 \mid b$ we can choose either value of $\theta^{2} / 3 \pm \theta$ for $\phi$. Set

$$
\varepsilon= \begin{cases}+1 & \text { if } 3 a-b \neq 0(\bmod 27), \\ -1 & \text { if } 3 a+b \neq \equiv(\bmod 27),\end{cases}
$$

subject to the remark above, so that

$$
\phi=\frac{\theta^{2}}{3}+\varepsilon \theta .
$$

The minimal polynomial of $\phi$ is

$$
p(x)=x^{3}-\frac{2 a}{3} x^{2}+\left(-a+\frac{a^{2}}{9}+\varepsilon b\right) x+\varepsilon b-\frac{b^{2}}{27}-\frac{\varepsilon a b}{9}
$$

so that $\phi \in O_{K}$. We have

$$
p(x) \equiv x^{3}-\frac{2 a}{3} x^{2}+\frac{a^{2}}{9} x \equiv x\left(x-\frac{a}{3}\right)^{2}(\bmod 3) .
$$

Further

$$
\operatorname{disc}(p(x))=\frac{D(\theta)(3 a-\varepsilon b-27)^{2}}{3^{6}} .
$$

As $3 \| \operatorname{disc}(p(x))$, $3 \| d(K)$, we have $3 \nmid \operatorname{ind}(\phi)$, so that by Theorem 1.1, we obtain

$$
\langle 3\rangle=\langle 3, \phi\rangle\left\langle 3, \phi-\frac{a}{3}\right\rangle^{2} .
$$


8 Decomposition of primes in a cubic field

\section{Case B4}

In this case we have $9 \mid a+1-b^{2}$. We set

$$
\phi= \begin{cases}\frac{\left(\theta^{2}-b \theta+1\right)}{3} & \text { if } 9 \| a+1-b^{2}, \\ \frac{\left(\theta^{2}+2 b \theta+1\right)}{3} & \text { if } 27 \mid a+1-b^{2} .\end{cases}
$$

First we consider the case $9 \| a+1-b^{2}$. The minimal polynomial of $\phi$ is

$$
p(x)=x^{3}-\frac{(2 a+3)}{3} x^{2}+\frac{(a+3)\left(a+1-b^{2}\right)}{9} x-\frac{\left(a+1-b^{2}\right)^{2}}{27}
$$

so that $p(x) \in \mathbb{Z}[x]$ and $\phi \in O_{K}$. We have

$$
p(x) \equiv x^{2}\left(x-\frac{2 a+3}{3}\right)(\bmod 3) .
$$

Further

$$
\operatorname{disc}(p(x))=b^{2} D(\theta) \frac{\left(a+1-b^{2}\right)^{2}}{3^{6}}
$$

so that $3\|\operatorname{disc}(p(x)), 3\| d(K)$, thus $3 \nmid \operatorname{ind}(\phi)$, and by Theorem 1.1 we have

$$
\langle 3\rangle=\langle 3, \phi\rangle^{2}\left\langle 3, \phi-\frac{2 a+3}{3}\right\rangle .
$$

Now we turn to the case $27 \mid a+1-b^{2}$. The minimal polynomial of $\phi$ is

$$
p(x)=x^{3}+p_{2} x^{2}+p_{1} x+p_{0},
$$

where

$$
\begin{gathered}
p_{2}=-\frac{(2 a+3)}{3}, \\
p_{1}=\frac{\left(a^{2}+4 a-4 a b^{2}+6 b^{2}+3\right)}{9}, \\
p_{0}=\frac{\left(-a^{2}-2 a+2 a b^{2}+8 b^{4}-7 b^{2}-1\right)}{27} .
\end{gathered}
$$

Clearly

$$
\begin{gathered}
p_{2} \in \mathbb{Z}, \\
p_{1}=(12 a-18)\left(\frac{a+1-b^{2}}{27}\right)-3\left(\frac{a}{3}\right)^{2}+2\left(\frac{a}{3}\right)+1 \in \mathbb{Z}, \\
p_{0}=\frac{a(a+1)}{3}+9\left(\frac{a+1-b^{2}}{27}\right)\left(24\left(\frac{a+1-b^{2}}{27}\right)-(2 a+1)\right) \in \mathbb{Z},
\end{gathered}
$$


Şaban Alaca et al. 9

so that $\phi \in O_{K}$. Further

$$
\begin{gathered}
p_{2} \equiv \frac{a}{3}+2(\bmod 3), \\
p_{1} \equiv \frac{2 a}{3}+1(\bmod 3), \\
p_{0} \equiv \frac{a}{3}(\bmod 3) .
\end{gathered}
$$

Hence

$$
p(x) \equiv\left(x+\frac{a}{3}\right)(x+1)^{2}(\bmod 3) .
$$

Further

$$
\operatorname{disc}(p(x))=b^{2} D(\theta) \frac{\left(8 b^{2}-2 a+1\right)^{2}}{3^{6}} .
$$

As $a \equiv 0,6(\bmod 9), a+1-b^{2} \equiv 0(\bmod 27)$, and

$$
8 b^{2}-2 a+1=6(a-3)-8\left(a+1-b^{2}\right)+27 ;
$$

we see that

$$
3^{2} \| 8 b^{2}-2 a+1
$$

so that $3\|\operatorname{disc}(p(x)), 3\| d(K)$, and thus $3 \nmid \operatorname{ind}(\phi)$. Hence by Theorem 1.1 we have

$$
\langle 3\rangle=\left\langle 3, \phi+\frac{a}{3}\right\rangle\langle 3, \phi+1\rangle^{2} .
$$

\section{Case B6}

In this case we set $\gamma_{3}(D(\theta))=2 m+3$ so that $m \geq 2$. Let

$$
\phi=\left\{\begin{array}{cc}
\frac{\lambda^{2}}{3}+\lambda & \text { if } a \neq 3^{m-1} D_{3}(\theta)(\bmod 9), \\
\frac{\lambda^{2}}{3}-\lambda & \text { if } a \neq \equiv-3^{m-1} D_{3}(\theta)(\bmod 9) .
\end{array}\right.
$$

The minimal polynomial of $\lambda$ is

$$
\begin{gathered}
p(x)=x^{3}-a D_{3}(\theta) x+3^{m} D_{3}(\theta)^{2}, \\
\operatorname{disc}(p(x))=3^{3} b^{2} D_{3}(\theta)^{3} .
\end{gathered}
$$


We are now in case B3 with

$$
\begin{gathered}
a^{\prime}=a D_{3}(\theta), \quad v_{3}\left(a^{\prime}\right)=1, \\
b^{\prime}=3^{m} D_{3}(\theta)^{2} \equiv 0(\bmod 9), \\
4 a^{\prime 3}-27 b^{\prime 2}=3^{3} b^{2} D_{3}(\theta)^{3}, \quad v_{3}\left(4 a^{\prime 3}-27 b^{\prime 2}\right)=3 .
\end{gathered}
$$

Hence

$$
\langle 3\rangle=\langle 3, \phi\rangle\left\langle 3, \phi-\frac{a D_{3}(\theta)}{3}\right\rangle^{2} .
$$

\section{Case B8}

Here $\langle 3\rangle$ is a prime ideal.

\section{Case C3}

Similarly to case B6 this case can be reduced to case C2.

\section{Cases C4, C5}

Here

$$
\begin{gathered}
p \nmid a, p \nmid b, \quad v_{p}(D(\theta)) \equiv 0(\bmod 2), \quad v_{p}(D(\theta)) \geq 2, \\
\left(\frac{D_{p}(\theta)}{p}\right)= \begin{cases}+1, & \text { case C4, } \\
-1, & \text { case C5. }\end{cases}
\end{gathered}
$$

Set $\nu_{p}(D(\theta))=2 m$ so that $m \geq 1$. Let $\phi=\alpha / p^{m}$. The minimal polynomial of $\phi$ is

$$
\begin{gathered}
p(x)=x^{3}-3 a D_{p}(\theta) x+p^{m} D_{p}(\theta)^{2}, \\
\operatorname{disc}(p(x))=\frac{3^{6} b^{2} D(\theta)^{3}}{p^{6 m}} .
\end{gathered}
$$

Clearly $p \nmid \operatorname{disc}(p(x))$ so that $p \nmid \operatorname{ind}(\phi)$. Now

$$
p(x) \equiv x\left(x^{2}-3 a D_{p}(\theta)\right)(\bmod p) .
$$

As

$$
4 a^{3}-27 b^{2} \equiv 0(\bmod p), \quad p \nmid a, p \nmid b, p>3,
$$

we have

$$
\left(\frac{3 a}{p}\right)=1
$$


Thus

$$
x^{2}-3 a D_{p}(\theta) \equiv \begin{cases}(x-t)(x+t)(\bmod p), & \text { case } \mathrm{C} 4 \\ \text { irreducible }(\bmod p), & \text { case C5 }\end{cases}
$$

where $t^{2} \equiv 3 a D_{p}(\theta)(\bmod p)$. Hence

$$
\langle p\rangle= \begin{cases}\langle p, \phi\rangle\langle p, \phi-t\rangle\langle p, \phi+t\rangle, & \text { case } \mathrm{C} 4, \\ \langle p, \phi\rangle\left\langle p, \phi^{2}-3 a D_{p}(\theta)\right\rangle, & \text { case } \mathrm{C} 5,\end{cases}
$$

where $N\left(\left\langle p, \phi^{2}-3 a D_{p}(\theta)\right\rangle\right)=p^{2}$.

\section{Acknowledgment}

The research of the second and third authors was supported by grants from the Natural Sciences and Engineering Research Council of Canada.

\section{References}

[1] Ş. Alaca, p-integral bases of a cubic field, Proceedings of the American Mathematical Society 126 (1998), no. 7, 1949-1953.

[2] Ş. Alaca, B. K. Spearman, and K. S. Williams, The factorization of 2 in cubic fields with index 2, Far East Journal of Mathematical Sciences (FJMS) 14 (2004), no. 3, 273-282.

[3] Ş. Alaca and K. S. Williams, Introductory Algebraic Number Theory, Cambridge University Press, Cambridge, 2004.

[4] H. Cohen, A Course in Computational Algebraic Number Theory, Springer, New York, 2000.

[5] B. N. Delone and D. K. Faddeev, The Theory of Irrationalities of the Third Degree, Translations of Mathematical Monographs, vol. 10, American Mathematical Society, Rhode Island, 1964.

[6] P. Llorente and E. Nart, Effective determination of the decomposition of the rational primes in a cubic field, Proceedings of the American Mathematical Society 87 (1983), no. 4, 579-585.

Şaban Alaca: Centre for Research in Algebra and Number Theory, School of Mathematics and Statistics, Carleton University Ottawa, ON, Canada K1S 5B6

E-mail address: salaca@math.carleton.ca

Blair K. Spearman: Department of Mathematics and Statistics, University of British Columbia Okanagan, Kelowna, BC, Canada V1V 1V7

E-mail address: blair.spearman@ubc.ca

Kenneth S. Williams: Centre for Research in Algebra and Number Theory, School of Mathematics and Statistics, Carleton University Ottawa, ON, Canada K1S 5B6

E-mail address: kwilliam@connect.carleton.ca 


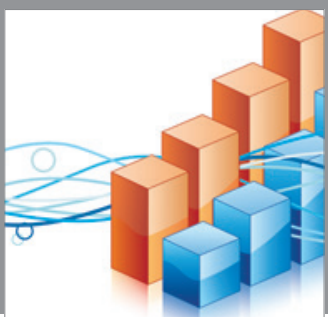

Advances in

Operations Research

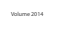

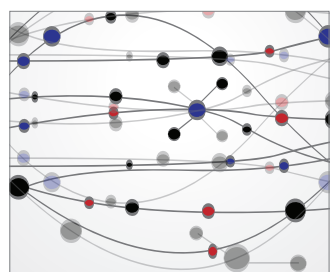

\section{The Scientific} World Journal
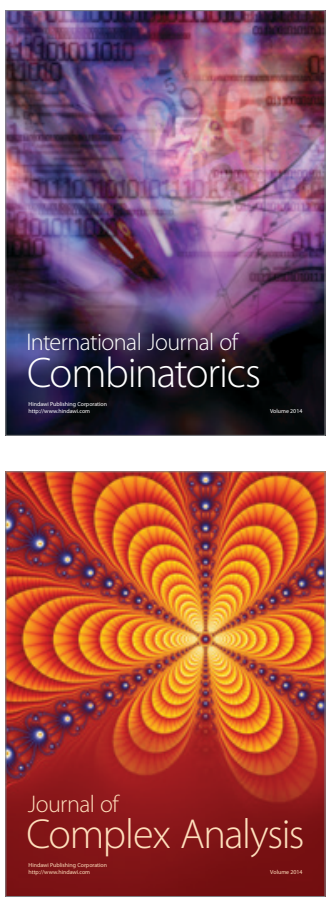

International Journal of

Mathematics and

Mathematical

Sciences
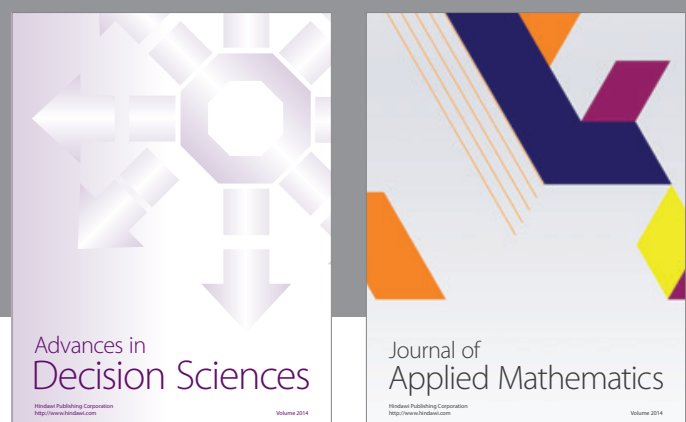

Journal of

Applied Mathematics
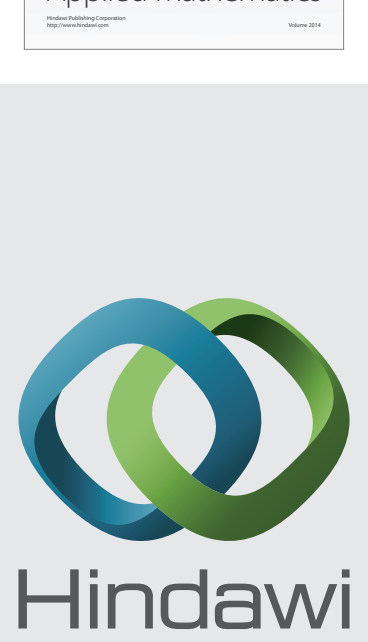

Submit your manuscripts at http://www.hindawi.com
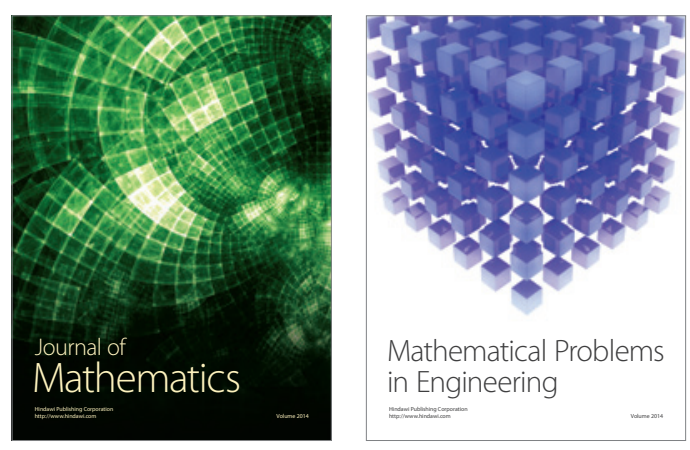

Mathematical Problems in Engineering
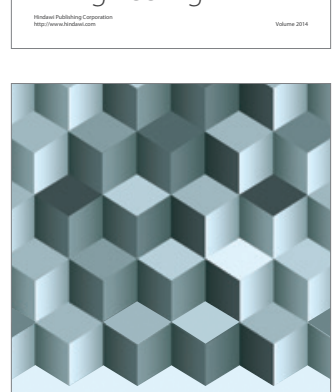

Journal of

Function Spaces
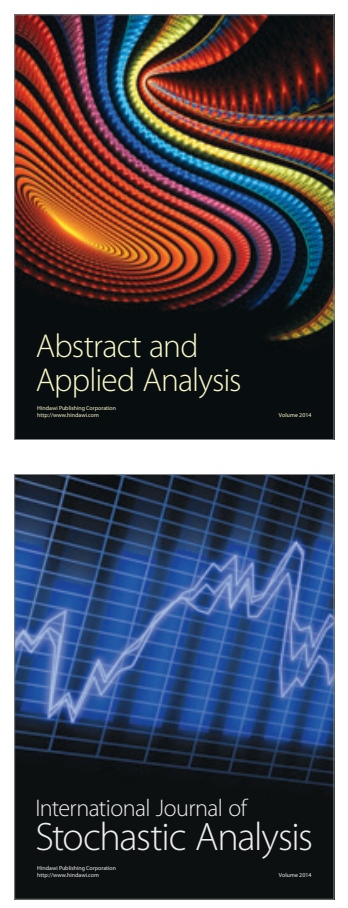

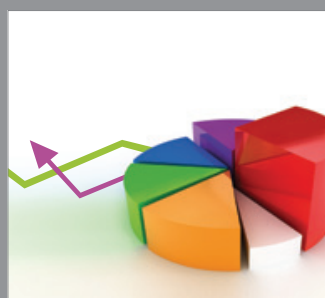

ournal of

Probability and Statistics

Promensencen
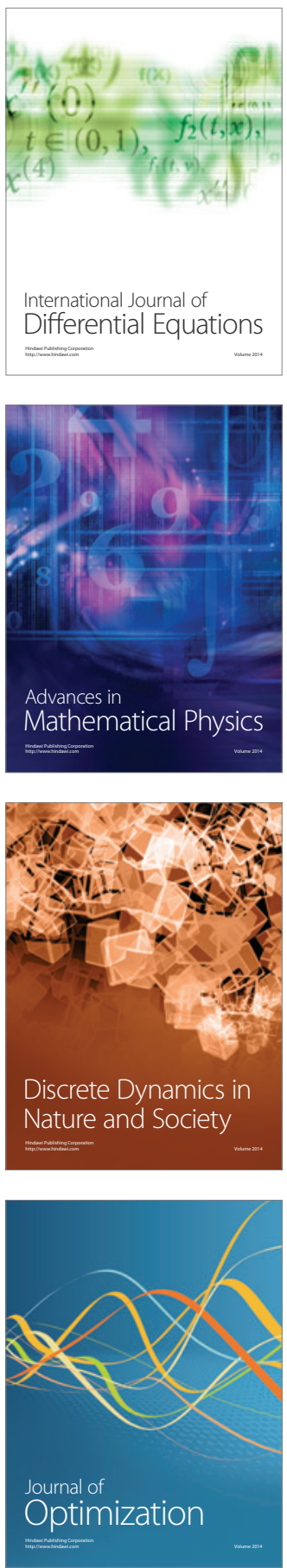